\title{
Estudo mecânico das propriedades dos fios de sutura usados em cirurgias ortopédicas*
}

\section{Mechanical Study of the Properties of Sutures Used in Orthopaedics Surgeries}

\author{
Leandro Cardoso Gomide ${ }^{1}$ Dagoberto de Oliveira Campos ${ }^{1}$ Cleudmar Amaral Araújo ${ }^{2}$ \\ Gabriela Lima Menegaz ${ }^{2}$ Rafael Silva Cardoso ${ }^{1}$ Sérgio Crosara Saad Júnior ${ }^{1}$

\footnotetext{
1 Departamento de Cirurgia, Faculdade de Medicina, Universidade Federal de Uberlândia, Uberlândia, MG, Brasil

2 Faculdade de Engenharia Mecânica, Universidade Federal de Uberlândia, Uberlândia, MG, Brasil
} \\ Address for correspondence Leandro Cardoso Gomide, Faculdade de \\ Medicina, Departamento de Cirurgia, Universidade Federal de \\ Uberlândia, Uberlândia, MG, Brasil \\ (e-mail: leandro@orthomedcenter.com.br).
}

Rev Bras Ortop 2019;54:247-252.

\author{
Resumo \\ Palavras-chave \\ - ombro \\ - articulação \\ - acromioclavicular/ \\ lesões \\ - articulação \\ - acromioclavicular/ \\ cirurgia \\ - fenômenos \\ biomecânicos \\ - suturas
}

Abstract
Objetivo Avaliar as propriedades mecânicas dos fios de sutura normalmente usados nas cirurgias ortopédicas e caracterizar seu comportamento por meio de ensaios de tração para verificar qual deles apresenta maior resistência mecânica.

Método Os ensaios de tração dos diferentes tipos de fios de sutura foram feitos na máquina de ensaios mecânicos BME 10 kN, com célula de carga de capacidade máxima de $50 \mathrm{kgf}$. Foram ensaiadas sete amostras de cada tipo de fio de sutura, foram fixadas cada uma das extremidades da amostra na garra metálica própria para o ensaio de fios e manteve-se o comprimento inicial de $5 \mathrm{~cm}$. Os ensaios foram feitos com uma velocidade de $20 \mathrm{~mm} /$ minuto e à temperatura ambiente, registraram-se os dados de força máxima e o deslocamento máximo na ruptura dos fios.

Resultados A força média de ruptura mais elevada foi observada na sutura FiberWire ${ }^{\circledR}$ 2 (Artrhex, Naples, FL, EUA) $\left(240,17 \mathrm{~N}\right.$ ), seguida pela HiFi ${ }^{\circledR} 2$ (Conmed, Utica, NY, EUA) $(213,39 \mathrm{~N})$ e Ethibond ${ }^{\circledR} 5$ (Ethicon Inc., Somerville, NJ, EUA) $(207,38 \mathrm{~N})$. A menor força média de ruptura foi obtida para o fio Ethibond ${ }^{\circledR} 2$ (Ethicon Inc., Somerville, NJ, EUA) $(97,8 \mathrm{~N})$.

Conclusão Os fios de sutura não absorvíveis de polimistura trançada, de surgimento mais recente, são superiores ao fio de sutura convencional de poliéster trançado. $\mathrm{O}$ FiberWire ${ }^{\circledR} 2$ é o mais resistente dos fios avaliados no presente estudo.

Objective To evaluate the mechanical properties of sutures commonly used in orthopedic surgeries, characterizing their behavior through tensile tests and determining which one has greater mechanical strength.

Estudo realizado na Faculdade de Medicina, Departamento de Cirurgia, Universidade Federal de Uberlândia, Uberlândia, MG, Brasil. Publicado originalmente por Elsevier Ltda.

received

July 8,2017

accepted

February 27, 2018
DOI https://doi.org/

10.1016/j.rbo.2018.02.001. ISSN 0102-3616.
Copyright $\odot 2019$ by Sociedade Brasileira License terms de Ortopedia e Traumatologia. Published by Thieme Revnter Publicações Ltda, Rio de Janeiro, Brazil 


\author{
Keywords \\ - shoulder \\ - acromioclavicular \\ joint/injuries \\ - acromioclavicular \\ joint/surgery \\ - biomechanical \\ phenomena \\ - sutures
}

Method Tensile tests of different sutures were performed in a mechanical testing machine BME $10 \mathrm{kN}$, using a $50 \mathrm{kgf}$ maximum capacity loading cell. Seven samples from each suture material were tested. Both ends of the sample material were fixed in the proper metal claw, maintaining an initial length of $5 \mathrm{~cm}$. Tests were performed at a speed of $20 \mathrm{~mm} /$ minute and at room temperature, recording data for maximum displacement and maximum force at the rupture point.

Results FiberWire ${ }^{\circledR} \# 2$ (Artrhex, Naples, FL, USA) presented the highest mean strength of rupture $(240.17 \mathrm{~N})$, followed by $\mathrm{HiFi}^{\circledR}$ \#2 (Conmed, Utica, NY, USA) (213.39N) and Ethibond ${ }^{\circledR} \# 5$ (Ethicon Inc., Somerville, NJ, USA) (207.38 N). Ethibond ${ }^{\circledR}$ \#2 (Ethicon Inc., Somerville, NJ, USA) had the lowestmean strength of rupture (97.8 N). Conclusion Non-absorbable braided polyblend sutures, more recently introduced, are superior to conventional, braided polyester sutures, and FiberWire ${ }^{\circledR} \# 2$ is the most resistant suture evaluated in the present study.

\section{Introdução}

Nas cirurgias ortopédicas, os principais objetivos são a restauração total da biomecânica ${ }^{1}$ e da anatomia. ${ }^{2}$ Para isso, existe grande variedade de material e métodos para tratamento de luxações e fraturas, como placas, parafusos, hastes intramedulares, pinos etc. Da mesma forma, também existem vários tipos e modelos de fios cirúrgicos para sutura e reparo de ligamentos. Como exemplo, podemos citar que a literatura recomenda uma variedade de procedimentos para o tratamento das lesões da articulação acromioclavicular que incluem fixação com pinos através da articulação acromioclavicular, transferência do ligamento coracoacromial pela técnica de Weaver-Dunn, fixação entre a clavícula e o processo coracoide, uso de placas, transferência muscular, amarrilho coracoclavicular com fios inabsorvíveis de grosso calibre, entre outros. ${ }^{3}$

Para que sejam usados fios de sutura com o intuito de manter a redução cirúrgica da articulação acromioclavicular, como, por exemplo, na técnica de amarrilho coracoclavicular, é necessário que eles tenham a capacidade de resistir às forças de tração a que normalmente são submetidos os ligamentos responsáveis pela estabilidade da articulação, em especial os coracoclaviculares, responsáveis pela estabilidade vertical da articulação. Além disso, o material ideal para a sutura deve ser de boa manipulação, ou seja, permitir um nó fácil, mínimo desconforto no dedo do cirurgião e boa capacidade de fixação. ${ }^{4} \mathrm{O}$ aspecto de resistência às forças de tração nos motivou a estudar as propriedades dos diversos fios de sutura e definir quais os melhores para serem usados em cirurgias ortopédicas.

O objetivo foi avaliar as propriedades dos fios de sutura normalmente usados em cirurgias ortopédicas e caracterizar seus comportamentos por meio de ensaios de tração, para verificar qual deles tem maior resistência mecânica.

\section{Material e métodos}

Este estudo avaliou o comportamento mecânico de quatro tipos de fios de sutura, comumente usados no tratamento cirúrgico da luxação acromioclavicular: Ethibond ${ }^{\circledR}$ no 2,
Ethibond ${ }^{\circledR}$ no 5, HiFi ${ }^{\circledR}$ no 2 e FiberWire ${ }^{\circledR}$ no 2 (- Fig. 1). Os fios Ethibond ${ }^{\circledR}$ n॰ 2 e Ethibond $^{\circledR}$ n॰ 5 são suturas não absorvíveis esterilizadas constituídas de poliéster, com construção multifilamentar tranc, ada e recobertos de polibutilato. A sutura FiberWire ${ }^{\circledR}$ no 2 é não absorvível e esterilizada, constituída por uma cadeia longa de multifilamentos com o núcleo de polietileno de peso molecular ultraelevado (UHMWPE) e revestida de poliéster entrançado. O fio de sutura $\mathrm{Hi}-\mathrm{Fi}^{\circledR}$ n。 2 é não absorvível, com estrutura trançada e sua composição também é baseada no polietileno de peso molecular ultraelevado.

Os ensaios de tração dos diferentes tipos de fios de sutura foram feitos na máquina de ensaios mecânicos BME $10 \mathrm{kN}$, com uma célula de carga de capacidade máxima de 50 kgf ( - Fig. 2). Foram ensaiadas sete amostras de cada tipo de fio de sutura, sem a presença do nó, fixou-se cada uma das extremidades da amostra na garra metálica própria para o ensaio de fios e manteve-se o comprimento inicial de $5 \mathrm{~cm}$ (-Fig. 3). Os ensaios foram feitos com uma velocidade de $20 \mathrm{~mm} / \mathrm{min}$ e à temperatura ambiente, registraram-se os dados de força máxima e deslocamento máximo na ruptura dos fios.

\section{Resultados}

Nos ensaios feitos foram obtidos os valores das forças máximas de ruptura ( $\mathrm{N}$ ), bem como a média deles. Também foram calculados os deslocamentos máximos na ruptura de cada fio $(\mathrm{mm})$, bem como a média, o fio Ethibond ${ }^{\circledR}$ no 2 apresentou uma força média de ruptura de 97,98 $\mathrm{N}$ e um deslocamento médio na ruptura de 35,24 mm (-Fig. 4). Já o fio Ethibond ${ }^{\circledR}$ n॰ 5 apresentou uma força média de ruptura de $207,38 \mathrm{~N}$ e um deslocamento médio na ruptura de $37,98 \mathrm{~mm}$ (-Fig. 5). Nos testes com a sutura $\mathrm{HiFi}^{\circledR}$ no 2 foi obtida uma força média de ruptura de $213,39 \mathrm{~N}$ e um deslocamento médio na ruptura de 72,90 mm (-Fig. 6). 0 maior valor de força de ruptura foi encontrado para o fio FiberWire $^{\circledR}$ no $2(240,17 \mathrm{~N})$, que obteve um deslocamento médio na ruptura de $34,19 \mathrm{~mm}$ (-Fig. 7). Os valores médios da força de ruptura obtidos nos ensaios foram comparados no gráfico mostrado na - Fig. 8. 


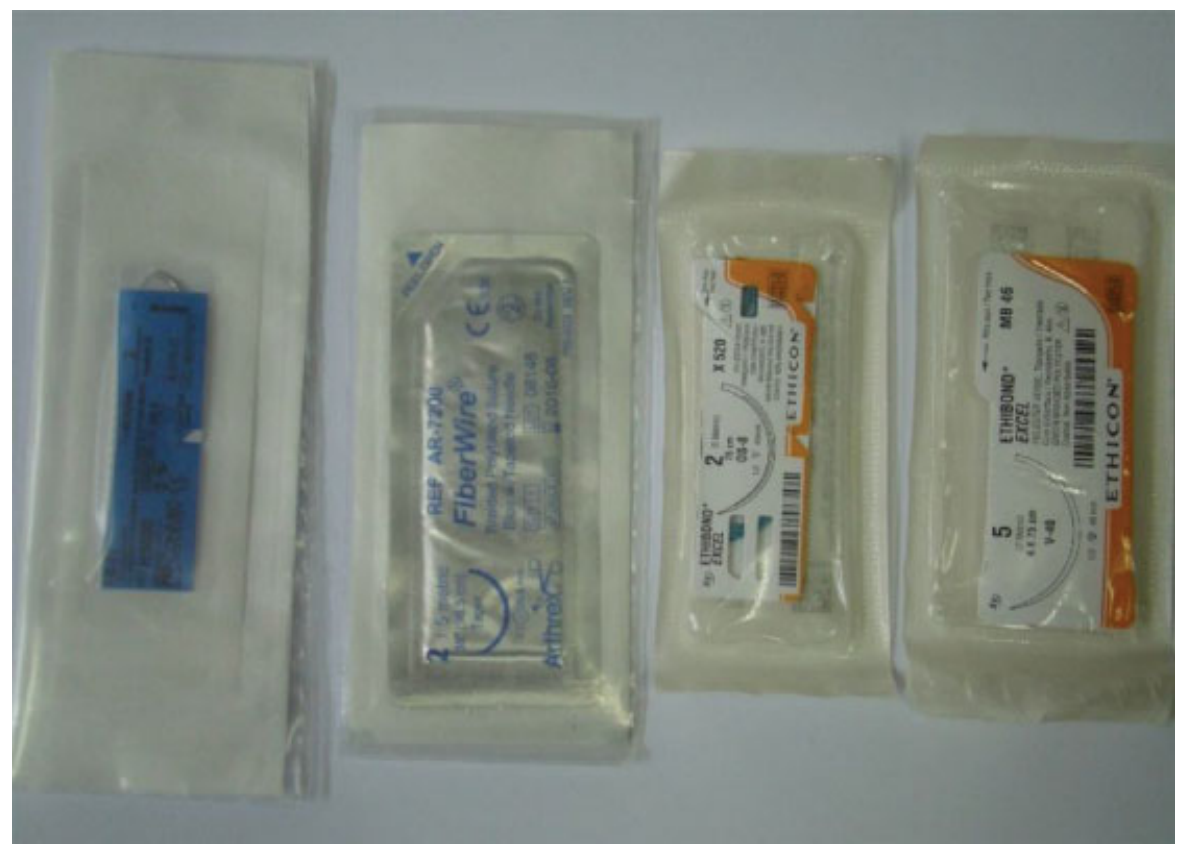

Fig. 1 Amostras dos fios de sutura HiFi ${ }^{\circledR}$ no 2, FiberWire ${ }^{\circledR}$ no 2, Ethibond ${ }^{\circledR}$ no 2 e Ethibond ${ }^{\circledR}$ no 5 .

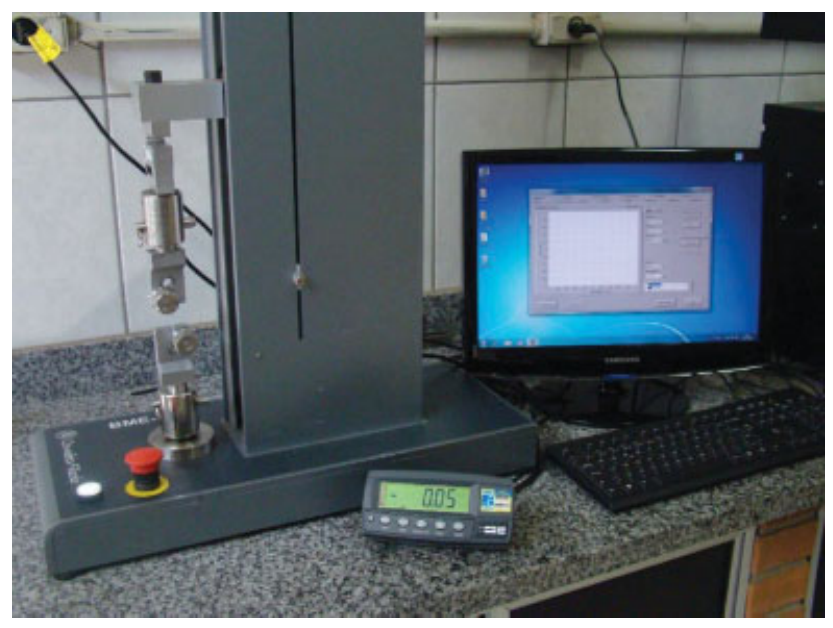

Fig. 2 Aparato experimental dos ensaios de tração: máquina de ensaios mecânicos BME 10 kN, célula de carga de 50 kgf e garras de fixação.

\section{Discussão}

Os fios de sutura são usados em cirurgias ortopédicas com a finalidade de fechamento de feridas, reparação de fáscia, músculos, tendões, ligamentos, cápsulas articulares e cerclagem ou banda de tensão de certas fraturas. A qualidade da reparação de tecidos depende de variáveis múltiplas, que incluem as características do tecido, as propriedades do material da sutura e a técnica cirúrgica usada. A escolha do material de sutura tem implicações importantes na reparação de tecidos, portanto resultados cirúrgicos adversos podem ser evitados pela seleção dele de acordo com a indicação diagnóstica. ${ }^{5}$

Tradicionalmente, no processo cirúrgico, eram usadas suturas de poliéster trançadas e não absorvíveis, pois esses tipos de sutura se mostravam mais resistentes e com menor tendência ao deslizamento do que as suturas de monofila-

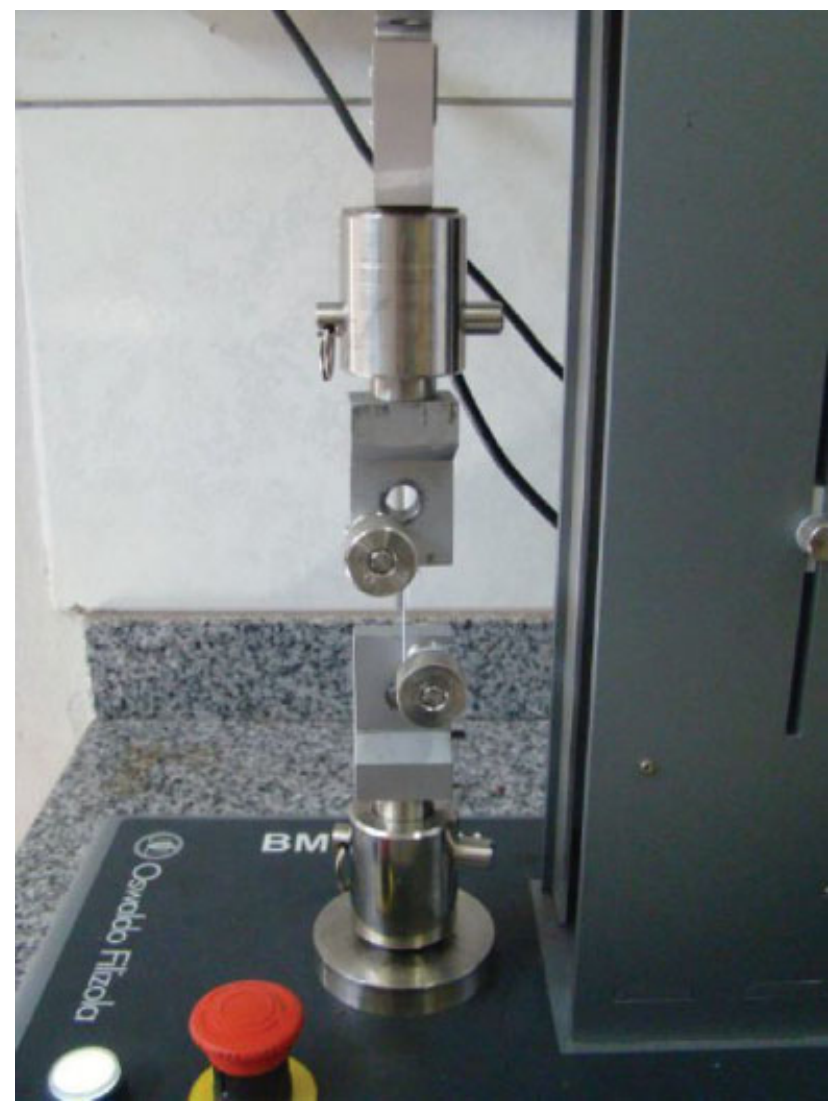

Fig.3 Garra metálica própria para o ensaio de fios com a manutenção do comprimento inicial de $5 \mathrm{~cm}$.

mento de polidiaxonona (PDS) absorvível. Entretanto, fatores como a frequente ruptura da sutura e a menor resistência conduziram ao desenvolvimento de suturas trançadas e não absorvíveis de uma polimistura, constituída de polietileno, poliéster e PDS. Assim, surgiram inúmeros fios de sutura com 


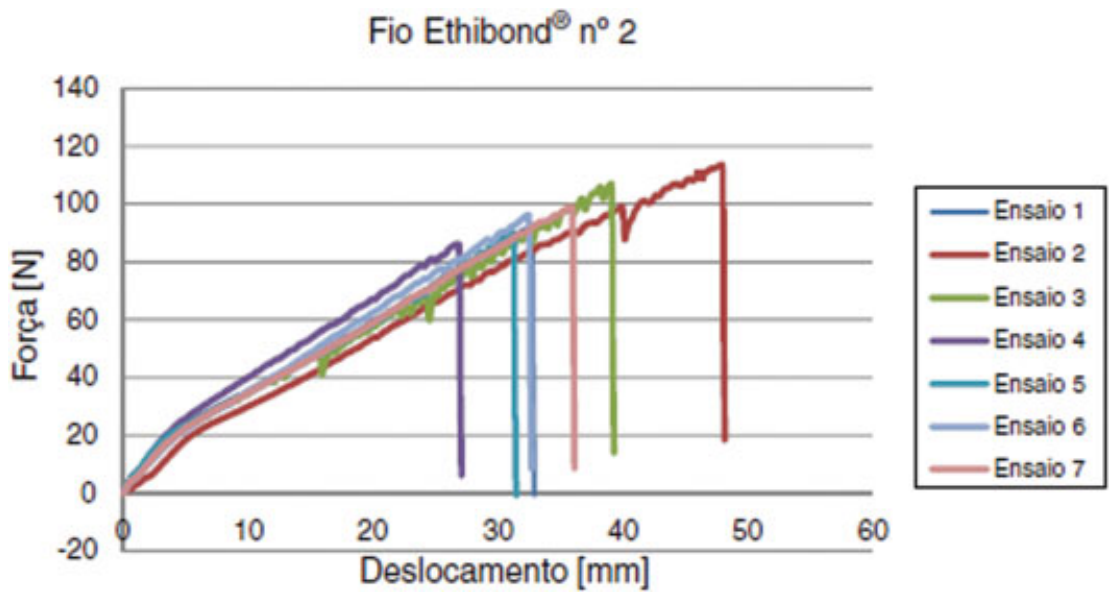

Fig. 4 Gráfico dos valores de força deformação dos ensaios do fio Ethibond ${ }^{\circledR}$ no 2 .

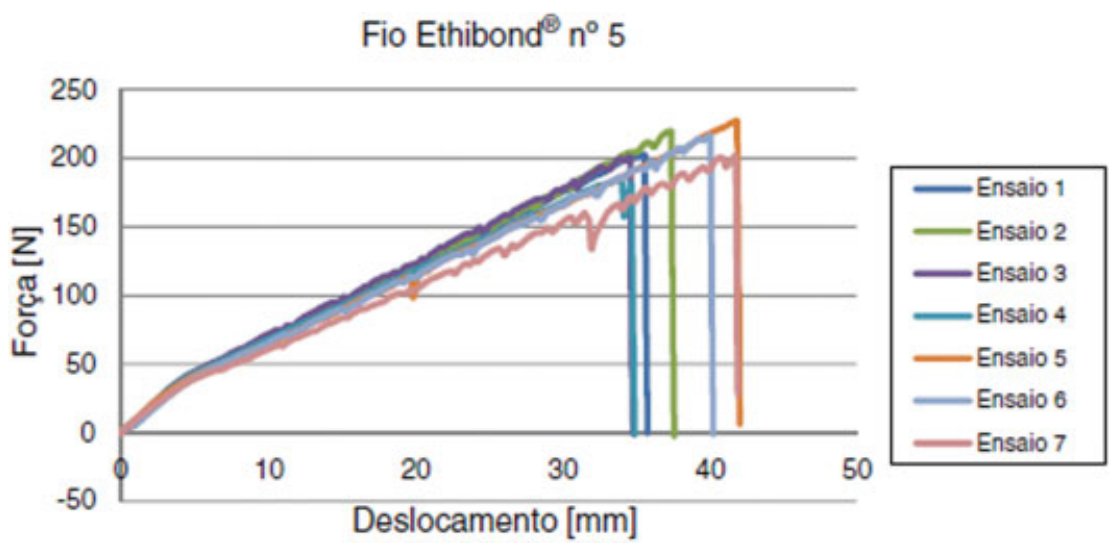

Fig. 5 Gráfico dos valores de força deformação dos ensaios do fio Ethibond ${ }^{\circledR}$ no 5 .

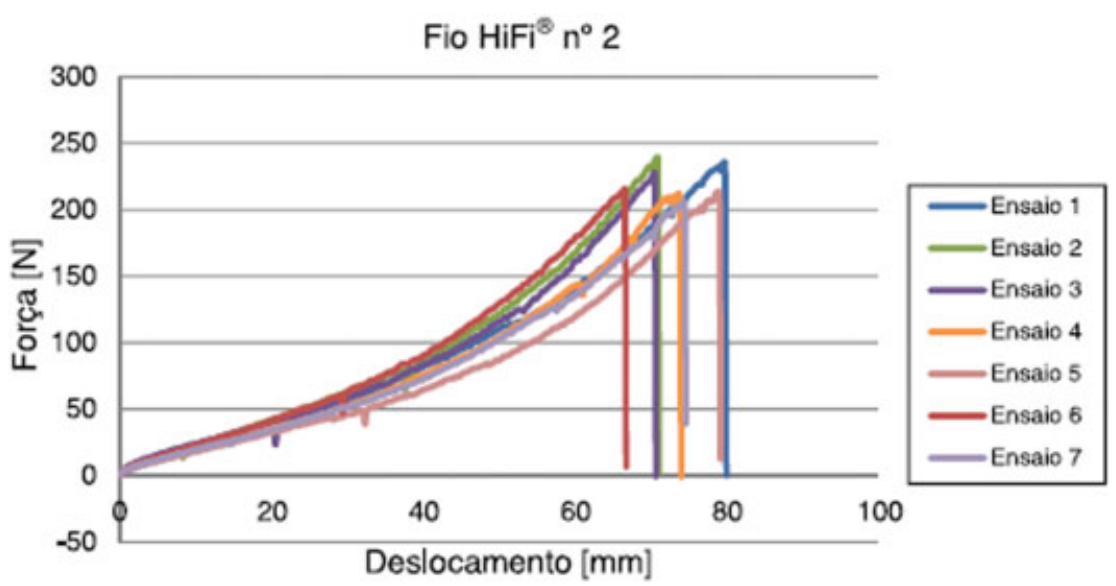

Fig. 6 Gráfico dos valores de força deformação dos ensaios do fio $\mathrm{HiFi}^{\circledR}$ no 2 .

essas características, como, por exemplo, os fios FiberWire ${ }^{\circledR} \mathrm{e}$ $\mathrm{HiFi}^{\circledR}, 8$ e seu uso tornou-se um procedimento comum em ortopedia.

Para que sejam usados fios de sutura com o intuito de manter a redução cirúrgica da articulação acromioclavicular, por exemplo, como na técnica de amarrilho coracoclavicular, eles devem ter a capacidade de resistir às forças de tração a que normalmente são submetidos os ligamentos envolvidos na estabilidade da articulação, em especial os coracoclaviculares, responsáveis pela estabilidade vertical. ${ }^{6}$

A literatura mostra que o ligamento coracoclavicular intacto suporta força de tração máxima de $500 \pm 134 \mathrm{~N}$, uma rigidez de $103 \pm 30 \mathrm{~N} / \mathrm{mm}$ e alongamento na ruptura de 7,7 $\pm 1,9$ mm, não há diferença significativa entre a contribuição do ligamento trapezoide e do conoide nessa configuração. O ligamento conoide isolado apresenta resistência 


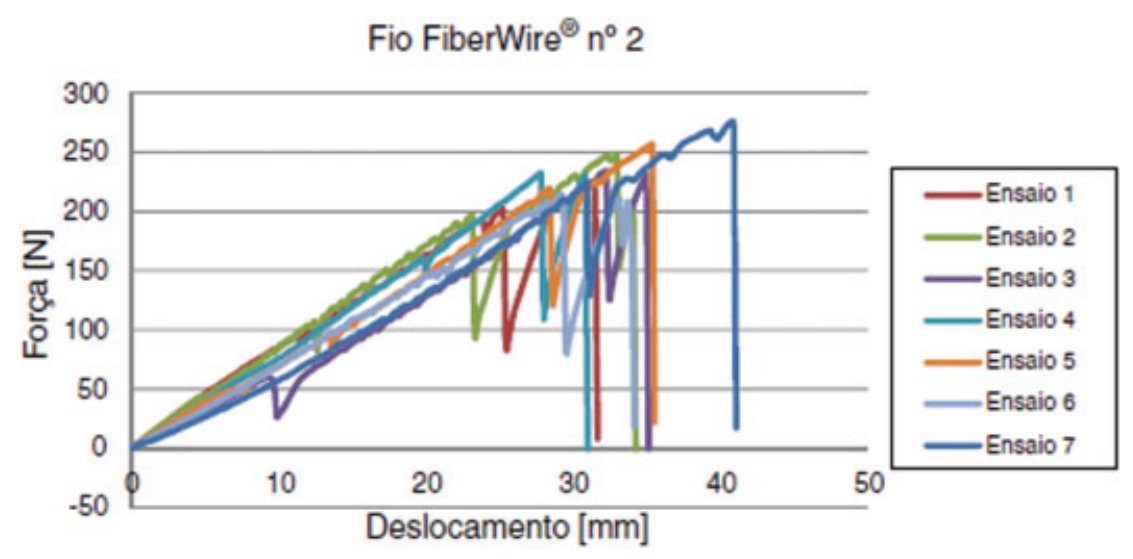

Fig. 7 Gráfico dos valores de força deformação dos ensaios do fio FiberWire ${ }^{\circledR}$ no 2 .

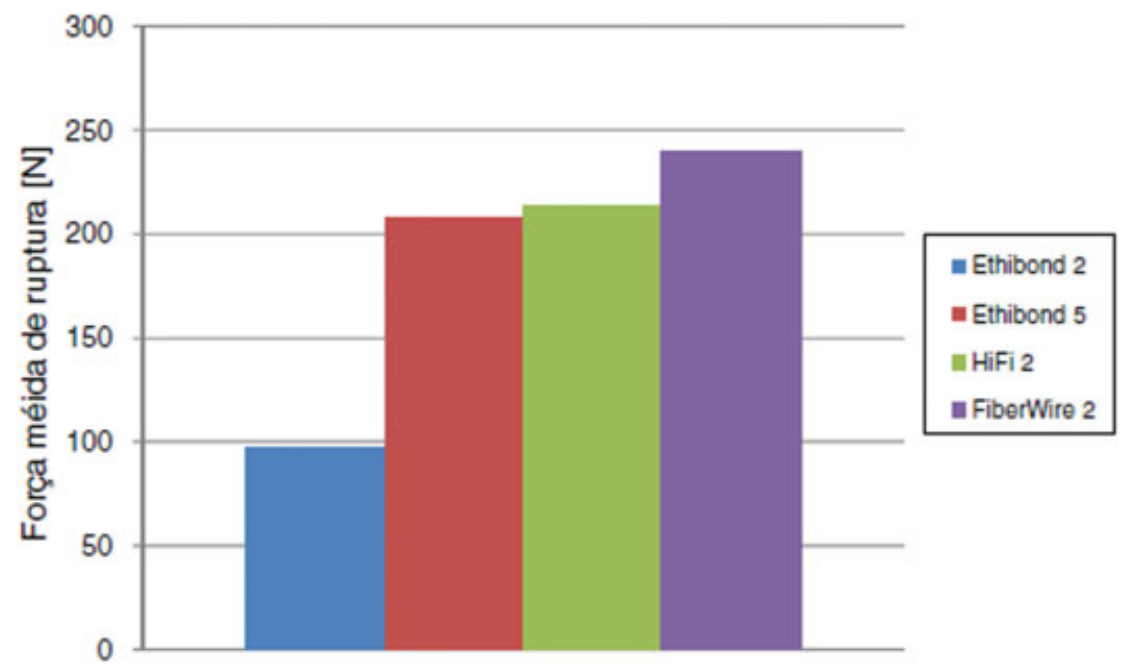

Fig. 8 Gráfico de comparação das forças médias de ruptura dos fios testados.

máxima de $394 \pm 170 \mathrm{~N}$, rigidez de $105 \pm 45 \mathrm{~N} / \mathrm{mm}$ e alongamento de $7,1 \pm 2,1 \mathrm{~mm}$. Para o ligamento trapezoide isolado foi encontrada a resistência máxima de $440 \pm 118 \mathrm{~N}$, rigidez de $84 \pm 18 \mathrm{~N} / \mathrm{mm}$ e alongamento de $9,2 \pm 2,6 \mathrm{~mm}^{7}$

Estudo feito por Wüst et $\mathrm{al}^{8}$ teve o objetivo de comparar as propriedades mecânicas das suturas não absorvíveis de polimistura trançada, FiberWire ${ }^{\circledR}, \mathrm{Hi}_{-} \mathrm{Fi}^{\circledR}$, Orthocord $^{\circledR}$ e Ultrabraid $^{\circledR}$, com a sutura convencional de poliéster trançado, Ethibond $^{\circledR}$, e as suturas absorvíveis de monofilamento de polidioxanona, PDS II e Ethicon ${ }^{\circledR}$, todas no 2. 0 trabalho teve como hipótese inicial que as suturas de polimistura são superiores às de poliéster trançado convencional e às de monofilamento degradável, em relação aos parâmetros de resistência à tração e alongamento máximo, mas requerem configurações de nó mais estáveis. Os ensaios foram feitos com a velocidade de $60 \mathrm{~mm} / \mathrm{min}$ e à temperatura ambiente. Comparados aos obtidos por Wüst et $\mathrm{al}^{8}{ }^{8}$ os resultados deste trabalho confirmaram que as suturas polimistura apresentam propriedades mecânicas vantajosas. Entretanto, os diferentes tipos de sutura polimistura têm diferenças significativas em suas propriedades. Todas as suturas polimistura testadas sem 0 nó foram de 2 a 2,5 vezes mais resistentes do que a sutura de poliéster e a de polidioxanona. Nos testes de resistência à tração das suturas sem nó, observou-se que o fio FiberWire ${ }^{\circledR}$ apresentou o maior valor de carga máxima de ruptura, $263 \mathrm{~N}$, e o fio Ethibond ${ }^{\circledR}$ apresentou força máxima de 110 N. Esses valores são condizentes com os obtidos no trabalho em questão: $240,17 \mathrm{~N}$ e $97,98 \mathrm{~N}$ para os fios FiberWire ${ }^{\circledR}$ e Ethibond $^{\circledR}$ no 2 , respectivamente. ${ }^{8}$

O trabalho de Wright et $\mathrm{al}^{9}$ estudou o comportamento das suturas não danificadas e danificadas testadas por ensaios de tração, além do desgaste das suturas que são passadas através das âncoras. A força de tração foi aplicada em um dos ensaios a 90 ॰ e em outro ensaio a 180。, no qual a sutura foi tracionada através do orifício da âncora de sutura. Foram testadas 20 amostras, 10 danificadas e 10 intactas, de cada sutura de no 2 de diferentes materiais: polidiaxanone (PDS), Ethibond ${ }^{\circledR}$, Tevdek $^{\circledR}$, Orthocord $^{\circledR}$ e FiberWire $^{\circledR}$, sempre em ambiente seco e a temperatura ambiente. A partir dos ensaios de tração para a ruptura da sutura foram determinados o módulo de elasticidade e a resistência à tração máxima. 0 teste de tração até a ruptura foi feito em uma máquina de ensaio MTS 858 Bionix, com velocidade de $60 \mathrm{~mm} / \mathrm{min}$. Para os fios não danificados, 0 FiberWire $^{\circledR}$ obteve os maiores valores de carga de ruptura máxima $(255,3 \pm 10,37 \mathrm{~N})$, seguido por Orthocord $^{\circledR}$ $(214,22 \pm 11,63 \mathrm{~N})$, PDS $(141,22 \pm 7,62 \mathrm{~N})$, Tevdek $^{\circledR}$ $(116,61 \pm 1,13 \mathrm{~N})$ e Ethibond ${ }^{\circledR}(114,58 \pm 1,58 \mathrm{~N})$. Esses 
valores estão em concordância com os obtidos neste estudo, que foram de $240,17 \mathrm{~N}$ e $97,98 \mathrm{~N}$, para os fios FiberWire $^{\circledR}$ e Ethibond ${ }^{\circledR}$ no 2, respectivamente. ${ }^{9}$

As propriedades biofísicas dos fios de sutura de alta resistência, FiberWire ${ }^{\circledR}$ n॰ 2 , $\operatorname{Orthocord}^{\circledR}$ n॰ $2, \mathrm{HiFi}^{\circledR}$ n॰ 2 e Ultrabraid ${ }^{\circledR}$ no 2, foram analisadas por microscopia óptica, microscopia eletrônica de varredura e testes mecânicos no trabalho de Jhamb et al. ${ }^{10}$ Os diferentes tipos de sutura foram carregados até a ruptura sem a presenc,a de nó em um dispositivo de teste Bionix 858 MTS, com uma célula de carga de $2 \mathrm{kN}$ para registrar as curvas de carga $\times$ deslocamento. As amostras ensaiadas tinham o comprimento de $5 \mathrm{~cm}$ e a velocidade usada no teste foi de $10 \mathrm{~mm} / \mathrm{min}$. A sutura Ultrabraid ${ }^{\circledR}$ obteve nos testes de tração a resistência máxima de $264 \mathrm{~N}$, seguida pela FiberWire ${ }^{\circledR} \operatorname{com} 238 \mathrm{~N}$, $\mathrm{HiFi}^{\circledR}$ com $215 \mathrm{~N}$ e Orthocord ${ }^{\circledR}$ com $212 \mathrm{~N}$. O valor obtido para a força de ruptura neste estudo para o fio FiberWire ${ }^{\circledR}$ foi de $240,17 \mathrm{~N}$ e para o fio $\mathrm{HiFi}^{\circledR}$ de $221,96 \mathrm{~N}$, estão de acordo com os valores apresentados no trabalho de Jhamb et al. ${ }^{10}$

A revisão destes trabalhos da literatura citados anteriormente mostrou diferentes velocidades adotadas para os ensaios de tração nos fios de sutura, que vão de $10 \mathrm{~mm} /$ min a $60 \mathrm{~mm} / \mathrm{min}$. A velocidade de $20 \mathrm{~mm} / \mathrm{min}$ está em um patamar entre esses valores e por isso foi a adotada por nós. Acreditamos que essa pequena diferença na velocidade não tem relevância nos resultados.

O estudo desenvolvido demonstrou superioridade dos fios da polimistura polietileno, poliéster e PDS (FiberWire ${ }^{\circledR}$ n॰ 2 e $\mathrm{HiFi}^{\circledR}$ n॰2) sobre os fios constituídos de poliéster, com construção multifilamentar trançada e recoberto de polibutilato (Ethibond ${ }^{\circledR}$ n॰ 5 e no 2 ). O FiberWire ${ }^{\circledR}$ n॰ 2 e o Hifi ${ }^{\circledR}$ no 2 apresentaram, respectivamente, força de ruptura média de $240,17 \mathrm{~N}$ e $213,38 \mathrm{~N}$; enquanto o fio Ethibond ${ }^{\circledR}$ no 5 resistiu a 207,38 N e o Ethibond ${ }^{\circledR}$ no 2 a 97,98 N.

Nenhum fio apresentou isoladamente uma resistência à tração superior à descrita na literatura para os ligamentos coracoclaviculares. Entretanto, o estudo não fez sutura semelhante aos amarrilhos coracoclaviculares feitos in vivo, e sim ensaios com amostras de fios isolados, fixados em garras metálicas. Por isso devem ser feitos novos estudos de tração com modelos biomecânicos que representem de forma simplificada a anatomia do ombro e os amarrilhos coracoclaviculares, para que uma melhor comparação entre a resistência dos ligamentos e dos amarrilhos com fios de sutura seja feita.

\section{Conclusão}

Os fios de sutura não absorvíveis de polimistura trançada, de surgimento mais recente, são superiores ao fio de sutura convencional de poliéster trançado. Dentre os fios testados, o mais resistente às forças de tração é o FiberWire ${ }^{\circledR}$ no 2 , seguido do $\mathrm{HiFi}^{\circledR}$ n॰ 2. Demonstraram-se menos resistentes os fios Ethibond ${ }^{\circledR}$ no 5 e Ethibond ${ }^{\circledR}$ no 2, o último foi o menos resistente.

\section{Conflitos de interesse}

Os autores declaram não haver conflitos de interesse.

\section{Referências}

1 Nordin M, Frankel VH. Biomecânica básica do sistema músculoesquelético. 3a ed. Rio de Janeiro: Guanabara Koogan; 2003

2 Moore KL, Dalley AF. Anatomia orientada para clínica. 4 a ed. Rio de Janeiro: Guanabara Koogan; 2005

3 Mazzocca AD, Santangelo SA, Johnson ST, Rios CG, Dumonski ML, Arciero RA. A biomechanical evaluation of an anatomical coracoclavicular ligament reconstruction. Am J Sports Med 2006;34 (02):236-246

4 Fukuda K, Craig EV, An KN, Cofield RH, Chao EY. Biomechanical study of the ligamentous system of the acromioclavicular joint. J Bone Joint Surg Am 1986;68(03):434-440

5 Najibi S, Banglmeier R, Matta J, Tannast M. Material properties of common suture materials in orthopaedic surgery. Iowa Orthop J 2010;30:84-88

6 Swan KG Jr, Baldini T, McCarty EC. Arthroscopic suture material and knot type: an updated biomechanical analysis. Am J Sports Med 2009;37(08):1578-1585

7 Harris RI, Wallace AL, Harper GD, Goldberg JA, Sonnabend DH, Walsh WR. Structural properties of the intact and the reconstructed coracoclavicular ligament complex. Am J Sports Med 2000;28(01):103-108

8 Wüst DM, Meyer DC, Favre P, Gerber C. Mechanical and handling properties of braided polyblend polyethylene sutures in comparison to braided polyester and monofilament polydioxanone sutures. Arthroscopy 2006;22(11):1146-53

9 Wright PB, Budoff JE, Yeh ML, Kelm ZS, Luo ZP. Strength of damaged suture: an in vitro study. Arthroscopy 2006;22(12): 1270-5.e3

10 Jhamb A, Goldberg J, Harper W, Butler A, Smitham PJ, Walsh WR. String theory: an examination of the properties of "high strength" suture materials. In: Annual Scientific Meeting of the Australian Orthopaedic Association; 2007 